\title{
Contribution to the Constrained Output Feedback Control
}

\author{
Souad Bezzaoucha, Benoît Marx, Didier Maquin, José Ragot
}

\begin{abstract}
In this paper, a Takagi-Sugeno model is used to represent the nonlinear behaviour of an actuator with saturation constraint. The control design is based on an output feedback controller (static or dynamic) depending on the saturation levels. Stabilization conditions are derived with the Lyapunov method and expressed in terms of linear matrix inequalities. Stabilisation conditions are addressed using a descriptor approach for the system modelling. An academic example is also presented with a comparison between different approaches.
\end{abstract}

Index Terms - Takagi-Sugeno models, nonlinear systems, actuator saturation, static and dynamic output feedback, linear matrix inequality, descriptor approach.

\section{INTRODUCTION}

Actuator saturation or control input saturation are probably the most usual nonlinearities encountered in control engineering because of the physical impossibility of applying unbounded control signals and/or safety constraints. Classical examples of such limits are the deflection limits in aircraft actuators, the voltage limits in electrical actuators and the limits on flow volume or rate in hydraulic actuators [12]. Motivated by these practical issues, many approaches have been developed to deal with actuator saturations in the existing literature (see, for example, [4], [5], [6], [12], [15] and the references therein).

The aim of this paper is to present another new treatment of saturation nonlinearity. A Takagi-Sugeno (T-S) formalism with sector nonlinearity approach is used to represent the nonlinear behaviour of a saturated actuator. This model, initially introduced in [9] is simple and accurate; it allows to use classical methods to study the nonlinear behaviors. The idea is to consider a set of linear sub-systems. An interpolation of all these sub-models with nonlinear functions satisfying the convex sum property allows to describe the global behavior of the system in a large operating range [11]. Our study is motivated by taking into account the saturation model during the controller design in order to obtain a controller which parameters depend on the saturation limits. The proposed representation describes the saturation as a T-S system with three sub-models and the weighting functions depend on the control input.

The design of stabilizing controllers for T-S nonlinear systems with input constraints can be addressed by using either an observer-based controller or an output feedback controller. The former approach requires additional dynamics and obsever design [11], [13]. The descriptor approach may also be envisaged [10], [2], [7] and [8]. The latter uses only measured

The authors are with Université de Lorraine, CRAN, UMR 7039, 2 avenue de la Forêt de Haye, Vandoeuvre-lès-Nancy Cedex, 54516, France. CNRS, CRAN, UMR 7039, France. firstname.nameduniv-lorraine. fr plant output signals [3], [14]. These controllers can be static or dynamic. Static output feedback control is the simplest approach since no further dynamics are needed. However, a dynamic compensator introducing extra dynamics is required to increase the number of degrees of freedom in the design and improve the closed-loop transient response.

In this paper, static and dynamic output feedback controllers are proposed. Sufficient linear matrix inequality (LMI) constraints are derived from the Lyapunov stability theory. Section II introduces the T-S structure for modeling, some preliminary results, mathematical notations and a brief description of the saturation. It is followed by the representation of the nonlinear saturation by a T-S structure in section III. In sections IV and V, static and dynamic output feedback controllers, depending on the saturation bounds, are respectively designed. In order to show the effectiveness of the proposed methods, some simulation results are given in section VI. Conclusions are detailed in section VII.

\section{Preliminaries}

The T-S modeling allows the representation of the behavior of nonlinear systems by the interpolation of a set of linear submodels. Each submodel contributes to the global behavior of the nonlinear system through a weighting function $h_{i}(\xi(t))$ [11]. The T-S structure is given by

$$
\left\{\begin{array}{l}
\dot{x}(t)=\sum_{i=1}^{n} h_{i}(\xi(t))\left(A_{i} x(t)+B_{i} u(t)\right) \\
y(t)=\sum_{i=1}^{n} h_{i}(\xi(t))\left(C_{i} x(t)+D_{i} u(t)\right)
\end{array}\right.
$$

where $x(t) \in \mathbb{R}^{n_{x}}$ is the system state, $u(t) \in \mathbb{R}^{n_{u}}$ the control input and $y(t) \in \mathbb{R}^{m}$ the system output. $\xi(t) \in \mathbb{R}^{q}$ is the decision variable assumed to be measurable (as the system output) or known (as the system input). The weighting functions $h_{i}(\xi(t))$ satisfy the so-called convex sum property

$$
\sum_{i=1}^{n} h_{i}(\xi(t))=1,0 \leq h_{i}(\xi(t)) \leq 1, \quad i=1, \ldots, n
$$

In the remaining of the paper, the following lemma is used.

Lemma 1: For any matrices $X, Y$ and $G=G^{T}>0$, the following inequality holds

$$
X^{T} Y+Y^{T} X \leq X^{T} G X+Y^{T} G^{-1} Y
$$

The following notations are used throughout the paper: a block diagonal matrix with the square matrices $A_{1}, \ldots, A_{n}$ on its diagonal is denoted $\operatorname{diag}\left(A_{1}, \ldots, A_{n}\right)$. The smallest and largest eigenvalues of the matrix $M$ are respectively denoted $\lambda_{\min }(M)$ and $\lambda_{\max }(M)$. The saturation function for 
a signal $v(t)$ is defined as (4), where $v_{\max }$ and $v_{\min }$ denote the saturation levels.

$$
\operatorname{sat}(v(t)):=\left\{\begin{array}{lll}
v(t) & \text { if } & v_{\min } \leq v(t) \leq v_{\max } \\
v_{\max } & \text { if } & v(t)>v_{\text {max }} \\
v_{\min } & \text { if } & v(t)<v_{\text {min }}
\end{array}\right.
$$

\section{PRoblem Statement}

A first contribution of this work is to model the nonlinear actuator saturation using the Takagi-Sugeno representation. For that, it is proposed to re-write the $j^{t h}$ component of the saturated control input (denoted $u_{s a t}^{j}(t)$, for $j=1, \ldots, n_{u}$ ) under a particular form of the $j^{t h}$ component of the control input (denoted $u_{j}(t)$, for $j=1, \ldots, n_{u}$ ), as follows:

$$
\begin{aligned}
u_{\text {sat }}^{j}(t) & =\operatorname{sat}\left(u_{j}(t)\right), \quad j=1, \ldots, n_{u} \\
& =\sum_{i=1}^{3} \mu_{i}^{j}(t)\left(\lambda_{i}^{j} u_{j}(t)+\gamma_{i}^{j}\right)
\end{aligned}
$$

with: $\lambda_{1}^{j}=0, \lambda_{2}^{j}=1, \lambda_{3}^{j}=0 ; \gamma_{1}^{j}=u_{\text {min }}^{j}, \gamma_{2}^{j}=0, \gamma_{3}^{j}=u_{\text {max }}^{j}$

and the weighting functions

$$
\left\{\begin{aligned}
\mu_{1}^{j}(t) & =\frac{1-\operatorname{sign}\left(u_{j}(t)-u_{\min }^{j}\right)}{2} \\
\mu_{2}^{j}(t) & =\frac{\operatorname{sign}\left(u_{j}(t)-u_{\min }^{j}\right)-\operatorname{sign}\left(u_{j}(t)-u_{\max }^{j}\right)}{2} \\
\mu_{3}^{j}(t) & =\frac{1+\operatorname{sign}\left(u_{j}(t)-u_{\max }^{j}\right)}{2}
\end{aligned}\right.
$$

Based on the convex sum property (2) of the weighting functions (7), equation (5) can be written in order to have the same activation functions for all the input vector components:

$$
u_{\text {sat }}^{j}(t)=\sum_{i=1}^{3} \mu_{i}^{j}(t)\left(\lambda_{i}^{j} u_{j}(t)+\gamma_{i}^{j}\right)\left(\prod_{\substack{\ell=1 \\ \ell \neq j}}^{n_{u}} \sum_{i=1}^{3} \mu_{i}^{\ell}(t)\right)
$$

For $n_{u}$ inputs, $3^{n_{u}}$ submodels are obtained. It is important to note that the saturated control is directly expressed in terms of the control variable $u(t)$. Equation (8) is equivalent to

$$
u_{\text {sat }}(t)=\sum_{i=1}^{3^{n_{u}}} \mu_{i}(t)\left(\Lambda_{i} u(t)+\Gamma_{i}\right)
$$

where the global weighting functions $\mu_{i}(t)$, the matrices $\Lambda_{i} \in$ $\mathbb{R}^{n_{u} \times n_{u}}$ and vectors $\Gamma_{i} \in \mathbb{R}^{n_{u} \times 1}$ are defined as follow

$$
\left\{\begin{aligned}
\mu_{i}(t) & =\prod_{j=1}^{n_{u}} \mu_{\sigma_{i}^{j}}^{j}\left(u_{j}(t)\right) \\
\Lambda_{i} & =\operatorname{diag}\left(\lambda_{\sigma_{i}^{1}}^{1}, \ldots, \lambda_{\sigma_{i}^{n_{u}}}^{n_{u_{u}}}\right) \\
\Gamma_{i} & =\left(\begin{array}{lll}
\gamma_{\sigma_{i}^{1}}^{1} & \ldots & \gamma_{\sigma_{i}^{n_{u}}}^{n_{u}}
\end{array}\right)^{T}
\end{aligned}\right.
$$

The indexes $\sigma_{i}^{j}\left(i=1, \ldots, 3^{n_{u}}\right.$ and $\left.j=1, \ldots, n_{u}\right)$, equal to 1,2 or 3 , indicate which partition of the $j^{\text {th }}$ input $\left(\mu_{1}^{j}, \mu_{2}^{j}\right.$ or $\mu_{3}^{j}$ ) is involved in the $i^{\text {th }}$ submodel.

The relations between the submodel number $i$ and the $\sigma_{i}^{j}$ indices are given by the following equation

$$
i=3^{n_{u}-1} \sigma_{i}^{1}+3^{n_{u}-2} \sigma_{i}^{2}+\ldots+3^{0} \sigma_{i}^{n_{u}}-\left(3^{1}+3^{2}+\ldots+3^{n_{u}-1}\right)
$$

For more details, see [1].
Let us now consider a T-S nonlinear system subject to actuator saturation represented by the following state equation

$$
\left\{\begin{array}{l}
\dot{x}(t)=\sum_{j=1}^{n} h_{j}(\xi(t))\left(A_{j} x(t)+B_{j} u_{s a t}(t)\right) \\
y(t)=\sum_{j=1}^{n} h_{j}(\xi(t))\left(C_{j} x(t)+D_{j} u_{s a t}(t)\right)
\end{array}\right.
$$

According to the T-S writting of the saturation developed above, equation (11) can be written as

$$
\left\{\begin{aligned}
\dot{x}(t) & =\sum_{i=1}^{3^{n_{u}}} \sum_{j=1}^{n} \mu_{i}(t) h_{j}(\xi(t))\left(A_{j} x(t)+B_{j}\left(\Lambda_{i} u(t)+\Gamma_{i}\right)\right) \\
y(t) & =\sum_{i=1}^{3^{n_{u}}} \sum_{j=1}^{n} \mu_{i}(t) h_{j}(\xi(t))\left(C_{j} x(t)+D_{j}\left(\Lambda_{i} u(t)+\Gamma_{i}\right)\right)
\end{aligned}\right.
$$

\section{Static OUtPut FeEdback CONTROLler: A DESCRIPTOR APPROACH}

The objective is to design a stabilizing static output feedback control ensuring the stability of the system, even in the presence of control input saturation. The solution is obtained by representing the saturation as a T-S system and by solving an optimization problem under LMI constraints. In order to highlight the interest of considering the saturation when computing the controller, the controller design is envisaged without (section IV-A) and with (section IV-B) a priori taking into account the input saturation. A comparaison of the obtained results is made in section VI.

\section{A. Nominal control law}

In order to stabilize the system at the origin, let us consider a linear output feedback controller given by (13)

$$
u(t)=\sum_{j=1}^{n} h_{j}(\xi(t)) K_{j}^{s} y(t)
$$

To reduce the number of LMIs to obtain the gains $K_{j}^{s}$, a descriptor approach is applied. This approach is well known to avoid the coupling terms between the feedback gains and the Lyapunov matrices. As a consequence, the number of LMI decreases and relaxed conditions are obtained [2]. The control law (13) and the output signal of system (1) are written as follows

$$
\left\{\begin{aligned}
0 . \dot{u}(t) & =\sum_{j=1}^{n} h_{j}(\xi(t)) K_{j}^{s} y(t)-u(t) \\
0 . \dot{y}(t) & =\sum_{j=1}^{n} h_{j}(\xi(t))\left(C_{j} x(t)+D_{j} u(t)\right)-y(t)
\end{aligned}\right.
$$

With the augmented state $x_{a}(t)=\left(\begin{array}{lll}x^{T}(t) & u^{T}(t) & y^{T}(t)\end{array}\right)^{T}$ and from equations (1) and (14), we can write

$$
E^{s} \dot{x}_{a}(t)=\sum_{j=1}^{n} h_{j}(\xi(t)) \mathscr{A}_{j}^{s} x_{a}(t)
$$

with

$$
E^{s}=\left(\begin{array}{ccc}
I_{n_{x}} & 0 & 0 \\
0 & 0 & 0 \\
0 & 0 & 0
\end{array}\right), \mathscr{A}_{j}^{s}=\left(\begin{array}{ccc}
A_{j} & B_{j} & 0 \\
0 & -I_{n_{u}} & K_{j}^{s} \\
C_{j} & D_{j} & -I_{m}
\end{array}\right)
$$


Let us consider the following Lyapunov function

$$
V(t)=x_{a}^{T}(t)\left(E^{s}\right)^{T} P x_{a}(t)
$$

with the condition

$$
\left(E^{s}\right)^{T} P=P^{T} E^{s} \geq 0
$$

and where the matrix $P$ is taken as $\operatorname{diag}\left(P_{1}, P_{2}, P_{3}\right)$ with $P_{1}=P_{1}^{T}, P_{2}>0$. One can note that $V(t)$ is quadratic in the system state, since (17) reduces to $V(t)=x^{T}(t) P_{1} x(t)$. The time derivative of the Lyapunov function is given by

$$
\dot{V}(t)=\sum_{j=1}^{n} h_{j}(\xi(t)) x_{a}^{T}(t)\left(\left(\mathscr{A}_{j}^{s}\right)^{T} P+P^{T} \mathscr{A}_{j}^{s}\right) x_{a}(t)
$$

To ensure the stability of (15), developing (19) with (16), the conditions to solve with regard to $P_{1}, P_{2}, P_{3}$ and $R_{j}$, $j=1, \ldots, n$ for the nominal case, are the following

$$
\left(\begin{array}{ccc}
A_{j}^{T} P_{1}+P_{1} A_{j} & P_{1} B_{j} & C_{j}^{T} P_{3} \\
* & -P_{2}-P_{2}^{T} & R_{j}+D_{j}^{T} P_{3} \\
* & * & -P_{3}-P_{3}^{T}
\end{array}\right)<0, j=1, \ldots, n
$$

The output feedback gains are deduced from $K_{j}=\left(P_{2}^{T}\right)^{-1} R_{j}$.

\section{B. Saturated T-S control law}

The objective is to adapt the gains $K_{j}$ of the controller (13) to the saturation limits and guarantee the stability of the closed loop system. The system (12) is written as a descriptor system using the augmented state $x_{a}(t)$ already defined

$$
E^{s} \dot{x}_{a}(t)=\sum_{i=1}^{3^{n_{u}}} \sum_{j=1}^{n} \mu_{i}(t) h_{j}(\xi(t))\left(\mathscr{A}_{i j}^{s} x_{a}(t)+\mathscr{B}_{i j}^{s}\right)
$$

with $E^{s}$ given by (16) and

$$
\mathscr{A}_{i j}^{s}=\left(\begin{array}{ccc}
A_{j} & B_{j} \Lambda_{i} & 0 \\
0 & -I_{n_{u}} & K_{j}^{s} \\
C_{j} & D_{j} \Lambda_{i} & -I_{m}
\end{array}\right), \mathscr{B}_{i j}^{S}=\left(\begin{array}{c}
B_{j} \Gamma_{i} \\
0 \\
D_{j} \Gamma_{i}
\end{array}\right)
$$

Theorem 1: There exists a static feedback controller (13) for a saturated input system (12) such that the system state converges toward an origin-centered ball of radius bounded by $\beta_{s}$ if there exists matrices $P_{1}^{s}=\left(P_{1}^{s}\right)^{T}>0, P_{2}^{s}>$ $0, P_{3}^{s}, R_{j}^{s}, \Sigma_{i j}^{1 s}=\left(\Sigma_{i j}^{1 s}\right)^{T}>0, \Sigma_{i j}^{3 s}=\left(\Sigma_{i j}^{3 s}\right)^{T}>0$, solutions of the following optimization problem (for $i=1, \ldots, 3^{n_{u}}$ and $j=1, \ldots, n)$

$$
\min _{1}^{s,}, P_{2}^{s}, P_{3}^{s}, R_{j}^{s}, \Sigma_{i j}^{1 s}, \Sigma_{i j}^{3 s} \beta_{s}
$$

s.t. (24)(see next page) with

$$
\Gamma_{i}^{T} B_{j}^{T} \Sigma_{i j}^{1 s} B_{j} \Gamma_{i}+\Gamma_{i}^{T} D_{j}^{T} \Sigma_{i j}^{3 s} D_{j} \Gamma_{i}<\beta_{s}
$$

The gains of the controller are given by $K_{j}^{s}=\left(\left(P_{2}^{s}\right)^{T}\right)^{-1} R_{j}^{s}$

Proof: Let us define the quadratic Lyapunov function

$$
V(t)=x_{a}^{T}(t)\left(E^{s}\right)^{T} P^{s} x_{a}(t)
$$

with the condition

$$
\left(E^{S}\right)^{T} P^{S}=\left(P^{S}\right)^{T} E^{s} \geq 0
$$

From (16) and (27), the matrix $P^{s}$ is chosen as follows, with $P_{1}^{s}=\left(P_{1}^{s}\right)^{T}>0$

$$
P^{s}=\operatorname{diag}\left(P_{1}^{s}, P_{2}^{s}, P_{3}^{s}\right)
$$

From equations (21) and (26), the time derivative of the Lyapunov function is given by

$$
\begin{gathered}
\dot{V}(t)=\sum_{i=1}^{3^{n_{u}}} \sum_{j=1}^{n} \mu_{i}(t) h_{j}(\xi(t))\left(\left(\mathscr{B}_{i j}^{s}\right)^{T} P^{s} x_{a}(t)\right. \\
\left.+x_{a}^{T}(t)\left(P^{s}\right)^{T} \mathscr{B}_{i j}^{S}+x_{a}^{T}(t)\left(\left(\mathscr{A}_{i j}^{s}\right)^{T} P^{s}+\left(P^{s}\right)^{T} \mathscr{A}_{i j}^{S}\right) x_{a}(t)\right)
\end{gathered}
$$

Using Lemma 1, it follows that

$$
\begin{gathered}
\left(\mathscr{B}_{i j}^{s}\right)^{T} P^{s} x_{a}(t)+x_{a}^{T}(t)\left(P^{s}\right)^{T} \mathscr{B}_{i j}^{s} \leq \\
\Gamma_{i}^{T} D_{j}^{T} \Sigma_{i j}^{3 s} D_{j} \Gamma_{i}+\Gamma_{i}^{T} B_{j}^{T} \Sigma_{i j}^{1 s} B_{j} \Gamma_{i} \\
+x_{a}^{T}(t) \operatorname{diag}\left(P_{1}^{s}\left(\Sigma_{i j}^{1 s}\right)^{-1} P_{1}^{s}, 0,\left(P_{3}^{s}\right)^{T}\left(\Sigma_{i j}^{3 s}\right)^{-1} P_{3}^{s}\right) x_{a}(t)
\end{gathered}
$$

Using (30), (29) is bounded as follows

$$
\begin{gathered}
\dot{V}(t) \leq \sum_{i=1}^{3^{n_{u}}} \sum_{j=1}^{n} \mu_{i}(t) h_{j}(\xi(t))\left(\Gamma_{i}^{T} B_{j}^{T} \Sigma_{i j}^{1 s} B_{j} \Gamma_{i}+\Gamma_{i}^{T} D_{j}^{T} \Sigma_{i j}^{3 s} D_{j} \Gamma_{i}\right. \\
\quad+x_{a}^{T}(t)\left(\left(\mathscr{A}_{i j}^{s}\right)^{T} P^{s}+\left(P^{s}\right)^{T} \mathscr{A}_{i j}^{s}+\right. \\
\left.\left.\quad \operatorname{diag}\left(P_{1}^{s}\left(\Sigma_{i j}^{1 s}\right)^{-1} P_{1}^{s}, 0, P_{3}^{s}\left(\Sigma_{i j}^{3 s}\right)^{-1} P_{3}^{s}\right)\right) x_{a}(t)\right)
\end{gathered}
$$

Let us define

$$
\begin{gathered}
\mathscr{Q}_{i j}^{s}=\left(\mathscr{A}_{i j}^{s}\right)^{T} P^{s}+\left(P^{s}\right)^{T} \mathscr{A}_{i j}^{s}+\operatorname{diag}\left(P_{1}^{s}\left(\Sigma_{i j}^{1 s}\right)^{-1} P_{1}^{s}, 0, P_{3}^{s}\left(\Sigma_{i j}^{3 s}\right)^{-1} P_{3}^{s}\right) \\
\varepsilon^{s}=\min _{i=1: 3^{n_{u}}, j=1: n} \lambda_{\min }\left(-\mathscr{Q}_{i j}^{s}\right) \\
\delta^{s}=\max _{i=1: 3^{n_{u}}, j=1: n}\left(\Gamma_{i}^{T} B_{j}^{T} \Sigma_{i j}^{1 s} B_{j} \Gamma_{i}+\Gamma_{i}^{T} D_{j}^{T} \Sigma_{i j}^{3 s} D_{j} \Gamma_{i}\right)
\end{gathered}
$$

Since $\Sigma_{i j}^{1 s}$ and $\Sigma_{i j}^{3 s}>0$, from equation (31) with the convex sum property (2), $\dot{V}(t)<-\varepsilon^{s}\left\|x_{a}\right\|_{2}^{2}+\delta^{s}$. It follows that $\dot{V}(t)<0$ for

$$
\mathscr{Q}_{i j}^{s}<0 \quad \text { and } \quad\left\|x_{a}\right\|_{2}^{2}>\frac{\delta^{s}}{\varepsilon^{s}}
$$

which means that $x_{a}(t)$ is uniformly bounded and converges to the origin-centered ball of radius $\sqrt{\frac{\delta^{s}}{\varepsilon^{s}}}$ according to Lyapunov stability theory [16].

The objective is now to minimize the radius $\sqrt{\frac{\delta^{s}}{\varepsilon^{s}}}$. Firstly $\delta^{s}$ (34) is bounded by $\beta_{s}$ from LMIs (25). Secondly, it can be shown that $1 / \varepsilon^{s}<\beta_{s}$. From (24), with a Schur's complement and the variable change

$$
R_{j}^{s}=\left(P_{2}^{s}\right)^{T} K_{j}^{s}
$$

it follows that

$$
-\mathscr{Q}_{i j}^{s}>\left(1 / \beta_{s}\right) I, i=1, \ldots, 3^{n_{u}}, j=1, \ldots, n
$$

meaning that all eigenvalues of $\left(-\mathscr{Q}_{i j}^{s}\right)$, including $\varepsilon^{s}$, are bigger then $1 / \beta_{s}$. Thus $1 / \mathcal{\varepsilon}^{s}<\beta_{s}$ and the radius $\sqrt{\frac{\delta^{s}}{\varepsilon^{s}}}$ is bounded by $\beta_{s}$.

Remark 1: It is important to highlight that the saturation may causes a performance degradation of the nonlinear system and even destabilize it. However, with the proposed 


$$
\left(\begin{array}{ccc|cc|ccc}
A_{j}^{T} P_{1}^{s}+P_{1}^{s} A_{j} & P_{1}^{s} B_{j} \Lambda_{i} & C_{j}^{T} P_{3}^{s} & P_{1}^{s} & 0 & I & 0 & 0 \\
* & -P_{2}^{s}-\left(P_{2}^{S}\right)^{T} & R_{j}^{s}+\Lambda_{i} D_{j}^{T} P_{3}^{s} & 0 & 0 & 0 & I & 0 \\
* & * & -P_{3}^{s}-\left(P_{3}^{S}\right)^{T} & 0 & P_{3}^{s} & 0 & 0 & I \\
\hline * & * & * & -\Sigma_{i j}^{1 s} & 0 & 0 & 0 & 0 \\
* & * & * & * & -\Sigma_{i j}^{3 s} & 0 & 0 & 0 \\
\hline * & * & * & * & * & -\beta_{s} I & 0 & 0 \\
* & * & * & * & * & * & -\beta_{s} I & 0 \\
* & * & * & * & * & * & * & -\beta_{s} I
\end{array}\right)<0
$$

static controller its stability is ensured.

Note that if the submodels are initially unstable, the proposed approach is not suitable for this case since the LMI condition can not be fulfiled. In order to improve the obtained results and relax the sabilization constraint, the dynamic output feedback control is proposed in the following section.

\section{DYNAMIC OUTPUT FEEDBACK CONTROLLER: A DESCRIPTOR APPROACH}

The objective is now to design a stabilizing dynamic output feedback control ensuring the stability of the system, even in the presence of control input saturation. The solution is obtained by representing the saturation as a T-S system and by solving an optimization problem under LMI constraints.

\section{A. Nominal control law}

In order to stabilize the system (1) at the origin, let us consider a dynamic output feedback controller defined by:

$$
\left\{\begin{array}{l}
\dot{x}_{c}(t)=\sum_{j=1}^{n} h_{j}(\xi(t))\left(A_{j}^{c} x_{c}(t)+B_{j}^{c} y(t)\right) \\
u(t)=\sum_{j=1}^{n} h_{j}(\xi(t))\left(C_{j}^{c} x_{c}(t)+D_{j}^{c} y(t)\right)
\end{array}\right.
$$

Once again, the descriptor approach is applied. Considering the augmented state vector $x_{a}(t)=$ $\left(\begin{array}{llll}x^{T}(t) & x_{c}^{T}(t) & u^{T}(t) & y^{T}(t)\end{array}\right)^{T}$, from equations (1) and (38), it follows

$$
E^{d} \dot{x}_{a}(t)=\sum_{j=1}^{n} h_{j}(\xi(t)) \mathscr{A}_{j}^{d} x_{a}(t)
$$

with $E^{d}=\operatorname{diag}\left(I_{2 n_{x}}, 0_{n_{u}+m}\right)$ and

$$
\mathscr{A}_{j}^{d}=\left(\begin{array}{cccc}
A_{j} & 0 & B_{j} & 0 \\
0 & A_{j}^{c} & 0 & B_{j}^{c} \\
0 & C_{j}^{c} & -I_{n_{u}} & D_{j}^{c} \\
C_{j} & 0 & D_{j} & -I_{m}
\end{array}\right)
$$

Let us consider the following Lyapunov function

$$
V(t)=x_{a}^{T}(t)\left(E^{d}\right)^{T} P x_{a}(t)
$$

with the condition

$$
\left(E^{d}\right)^{T} P=P^{T} E^{d} \geq 0
$$

From (42) and (40), the matrix $P$ is chosen as

$$
P=\left(\begin{array}{cccc}
P_{11} & 0 & 0 & 0 \\
0 & P_{22} & 0 & 0 \\
0 & 0 & P_{33} & 0 \\
P_{41} & P_{42} & P_{43} & P_{44}
\end{array}\right)
$$

with

$$
P_{11}=P_{11}^{T}>0, P_{22}=P_{22}^{T}>0, P_{33}>0
$$

The time derivative of the Lyapunov function is given by

$$
\dot{V}(t)=\sum_{j=1}^{n} h_{j}(\xi(t)) x_{a}^{T}(t)\left(\left(\mathscr{A}^{d}\right)_{j}^{T} P+P^{T} \mathscr{A}_{j}^{d}\right) x_{a}(t)
$$

To ensure the stability of (39), the conditions to satisfy, for the nominal case, are given by (46) (for $j=1, \ldots, n$ ) with

$$
\left\{\begin{aligned}
A_{j}^{c} & =P_{22}^{-1} \bar{A}_{j}^{c} & B_{j}^{c} & =P_{22}^{-1} \bar{B}_{j}^{c} \\
C_{j}^{c} & =\left(P_{33}^{T}\right)^{-1} \bar{C}_{j}^{c} & D_{j}^{c} & =\left(P_{33}^{T}\right)^{-1} \bar{D}_{j}^{c}
\end{aligned}\right.
$$

\section{B. Saturated T-S control law}

The objective is now to design the dynamic controller (38) to guarantee the stability of the saturated system (12) according to the saturation limits. Considering the previous augmented state vector $x_{a}(t)$, from equations (12) and (38), the closed loop system is given by:

$$
E^{d} \dot{x}_{a}(t)=\sum_{i=1}^{3^{n_{u}}} \sum_{j=1}^{n} \mu_{i}(t) h_{j}(\xi(t))\left(\mathscr{A}_{i j}^{d} x_{a}(t)+\mathscr{B}_{i j}^{d}\right)
$$

with $E^{d}=\operatorname{diag}\left(I_{2 n_{x}}, 0_{n_{u}+m}\right)$ and

$$
\mathscr{A}_{i j}^{d}=\left(\begin{array}{cccc}
A_{j} & 0 & B_{j} \Lambda_{i} & 0 \\
0 & A_{j}^{c} & 0 & B_{j}^{c} \\
0 & C_{j}^{c} & -I_{n_{u}} & D_{j}^{c} \\
C_{j} & 0 & D_{j} \Lambda_{i} & -I_{m}
\end{array}\right), \mathscr{B}_{i j}^{d}=\left(\begin{array}{c}
B_{j} \Gamma_{i} \\
0 \\
0 \\
D_{j} \Gamma_{i}
\end{array}\right)
$$

Theorem 2: There exists a dynamic feedback controller (38) for a saturated input system (12) such that the system state converges toward an origin-centered ball of radius bounded by $\beta_{d}$ if there exists $P_{11}^{d}=\left(P_{11}^{d}\right)^{T}>0, P_{22}^{d}=$ $\left(P_{22}^{d}\right)^{T}>0, P_{33}^{d}>0, P_{41}^{d}, P_{42}^{d}, P_{43}^{d}, P_{44}^{d}, \bar{A}_{j}^{c}, \bar{B}_{j}^{c}, \bar{C}_{j}^{c}, \bar{D}_{j}^{c}, \Sigma_{i j}^{1 d}=$ $\left(\Sigma_{i j}^{1 d}\right)^{T}>0$, and $\Sigma_{i j}^{2 d}=\left(\Sigma_{i j}^{2 d}\right)^{T}>0$, solutions of the following optimization problem (for $i=1, \ldots, 3^{n_{u}}$ and $j=1, \ldots, n$ )

$$
P_{11}^{d}, P_{22}^{d}, P_{33}^{d}, P_{41}^{d}, P_{42}^{d}, P_{43}^{d}, P_{44}^{d}, \bar{A}_{j}^{c}, \bar{B}_{j}^{c}, \bar{C}_{j}^{c}, \bar{D}_{j}^{c}, \Sigma_{i j}^{1 d}, \Sigma_{i j}^{2 d} \beta_{d}
$$

s.t. (50) (see next page) with

$$
\Gamma_{i}^{T} B_{j}^{T} \Sigma_{i j}^{1 d} B_{j} \Gamma_{i}+\Gamma_{i}^{T} D_{j}^{T} \Sigma_{i j}^{2 d} D_{j} \Gamma_{i}<\beta_{d}
$$

The gains of the controller (38) are given by

$$
\left\{\begin{aligned}
A_{j}^{c} & =\left(P_{22}^{d}\right)^{-1} \bar{A}_{j}^{c} & B_{j}^{c} & =\left(P_{22}^{d}\right)^{-1} \bar{B}_{j}^{c} \\
C_{j}^{c} & =\left(\left(P_{33}^{d}\right)^{-1}\right)^{T} \bar{C}_{j}^{c} & D_{j}^{c} & =\left(\left(P_{33}^{d}\right)^{-1}\right)^{T} \bar{D}_{j}^{c}
\end{aligned}\right.
$$




$$
\left(\begin{array}{cccc}
A_{j}^{T} P_{11}+P_{11} A_{j}+C_{j}^{T} P_{41}+P_{41}^{T} C_{j} & C_{j}^{T} P_{42} & P_{11} B_{j}+C_{j}^{T} P_{43}+P_{41}^{T} D_{j} & C_{j}^{T} P_{44}-P_{41}^{T} \\
* & \left(\bar{A}_{j}^{c}\right)^{T}+\bar{A}_{j}^{c} & \left(\bar{C}_{j}^{c}\right)^{T}+P_{42}^{T} D_{j} & \bar{B}_{j}^{c}-P_{42}^{T} \\
* & * & P_{43}^{T} D_{j}+\left(P_{43}^{T} D_{j}\right)^{T}-P_{33}-P_{33}^{T} & \bar{D}_{j}^{c}-P_{43}^{T}+D_{j}^{T} P_{44} \\
* & * & * & -P_{44}-P_{44}^{T}
\end{array}\right)<0
$$

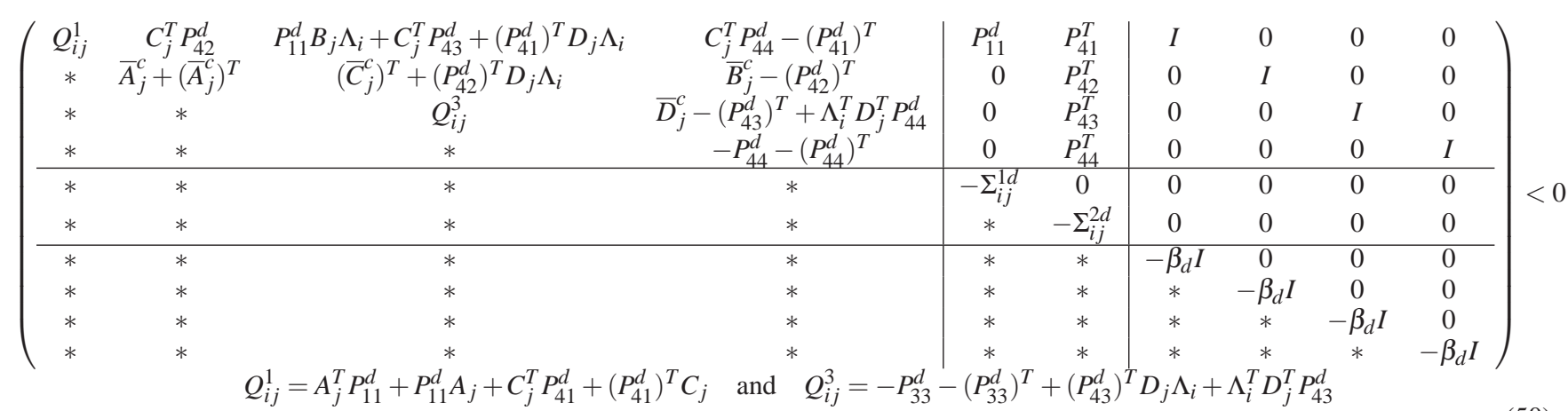

Proof: Let us define the Lyapunov function $V(t)=$ $x_{a}^{T}(t)\left(E^{d}\right)^{T} P^{d} x_{a}(t)$ with $E^{d}$ defined as (40) and the condition $\left(E^{d}\right)^{T} P^{d}=\left(P^{d}\right)^{T} E^{d} \geq 0$.

To satisfy this condition, the matrix $P^{d}$ is then chosen

$$
P^{d}=\left(\begin{array}{cccc}
P_{11}^{d} & 0 & 0 & 0 \\
0 & P_{22}^{d} & 0 & 0 \\
0 & 0 & P_{33}^{d} & 0 \\
P_{41}^{d} & P_{42}^{d} & P_{43}^{d} & P_{44}^{d}
\end{array}\right)
$$

with $P_{11}^{d}=\left(P_{11}^{d}\right)^{T}>0, P_{22}^{d}=\left(P_{22}^{d}\right)^{T}>0$ and $P_{33}^{d}>0$. Applying the same development as for the static controller with the variable changes

$$
\left\{\begin{array}{lll}
\bar{A}_{j}^{c}=P_{22}^{d} A_{j}^{c} & \bar{B}_{j}^{c}=P_{22}^{d} B_{j}^{c} \\
\bar{C}_{j}^{c}=\left(P_{33}^{d}\right)^{T} C_{j}^{c} & \bar{D}_{j}^{c}=\left(P_{33}^{d}\right)^{T} D_{j}^{c}
\end{array}\right.
$$

and defining $\varepsilon^{d}$ and $\delta^{d}$ by

$$
\begin{aligned}
& \varepsilon^{d}=\min _{i=1: 3^{n_{u}}, j=1: n} \lambda_{\min }\left(-\mathscr{Q}_{i j}^{d}\right) \\
& \delta^{d}=\max _{i=1: 3^{n_{u}}, j=1: n} \Gamma_{i}^{T}\left(B_{j}^{T} \Sigma_{i j}^{1 d} B_{j}+D_{j}^{T} \Sigma_{i j}^{2 d} D_{j}\right) \Gamma_{i}
\end{aligned}
$$

with $\mathscr{Q}_{i j}^{d}$ defined in the same way as $\mathscr{Q}_{i j}^{s}$ was.

The stabilizing conditions are linearized and given by (50). As the weighting functions satisfy (2) and $\Sigma_{i j}^{1 d}, \Sigma_{i j}^{2 d}>0$, if (50) holds, and $\left\|x_{a}\right\|_{2}^{2}>\frac{\delta^{d}}{\varepsilon^{d}}$, then $\dot{V}(x(t))<0$, implying that $x_{a}(t)$ converges to an origin centered ball of radius $\sqrt{\frac{\delta^{d}}{\varepsilon^{d}}}$. Similarily to what is done in the proof of theorem 1 , the radius of the ball is bounded by $\beta^{d}$ due to (50) and (49).

\section{NumERICAL EXAMPLE}

This section is devoted to the comparison of the results obtained with the nominal controller and with the proposed controller, both in the static (13) and dynamic cases (38). Let us consider system (11) with $n=2, D_{1}=D_{2}=\left(\begin{array}{l}0 \\ 0\end{array}\right)$ and

$$
\begin{aligned}
& A_{1}=\left(\begin{array}{ccc}
-2 & 1 & 1 \\
1 & -3 & 0 \\
2 & 1 & -8
\end{array}\right), A_{2}=\left(\begin{array}{ccc}
-3 & 2 & -2 \\
5 & -3 & 0 \\
1 & 2 & -4
\end{array}\right) \\
& B_{1}=\left(\begin{array}{c}
1 \\
5 \\
1
\end{array}\right), B_{2}=\left(\begin{array}{c}
3 \\
1 \\
-1
\end{array}\right), C_{1}=C_{2}=\left(\begin{array}{ccc}
1 & 1 & 1 \\
1 & 0 & 0
\end{array}\right)
\end{aligned}
$$

The input is subject to the following saturations $u_{\max }=$ $0.3, u_{\min }=-0.3$. The weighting functions depend on the output and are defined as follows

$\mu_{1}(x(t))=\frac{\left(1-\tanh \left(y_{1}(t)+y_{2}(t)\right)\right)}{2} ; \mu_{2}(x(t))=1-\mu_{1}(x(t))$

The figures 3 and 4 depict the system outputs and the control input of the nominal closed loop system without saturation (respectively denoted $y_{1 n}, y_{2 n}$ and $u_{n}$ ), those of the nominal closed loop system with saturation (respectively denoted $y_{1 s a t}, y_{2 s a t}$ and $u_{n s a t}$ ), those obtained by the static T-S controller (respectively denoted $y_{1}^{s}, y_{2}^{s}$ and $u^{s}$ ) and those obtained with the dynamic T-S controller (respectively denoted $y_{1}^{d}, y_{2}^{d}$ and $u^{d}$ ). For this example, the nominal controller is

$$
K_{1}^{n}=\left(\begin{array}{ll}
0.05 & 0.12
\end{array}\right), K_{2}^{n}=\left(\begin{array}{ll}
-0.13 & -0.18
\end{array}\right)
$$

The static controller gains from Theorem 1 are equal to

$$
K_{1}^{s}=\left(\begin{array}{ll}
0.03 & 0.06
\end{array}\right), K_{2}^{s}=\left(\begin{array}{ll}
0.03 & -0.43
\end{array}\right)
$$

The dynamic controller from Theorem 2 is given by

$$
\begin{aligned}
& A_{1}^{c}=\left(\begin{array}{ccc}
-11.34 & -9.11 & -9.11 \\
-9.11 & -11.34 & -9.11 \\
-9.11 & -9.11 & -11.34 \\
-2.31 & -0.08 & -0.08 \\
-0.08 & -2.31 & -0.08 \\
-0.08 & -0.08 & -2.31
\end{array}\right),\left(C_{1}^{c}\right)^{T}=\left(\begin{array}{l}
-0.56 \\
-0.56 \\
-0.56
\end{array}\right) \\
& A_{2}^{c}=\left(\begin{array}{l}
-0.31 \\
-0.31 \\
-0.31
\end{array}\right) \\
& B_{1}^{c}=\left(\begin{array}{ll}
-0.15 & -0.16 \\
-0.15 & -0.16 \\
-0.15 & -0.16
\end{array}\right), B_{2}^{c}=\left(\begin{array}{ll}
-0.068 & -0.029 \\
-0.068 & -0.029 \\
-0.068 & -0.029
\end{array}\right)
\end{aligned}
$$



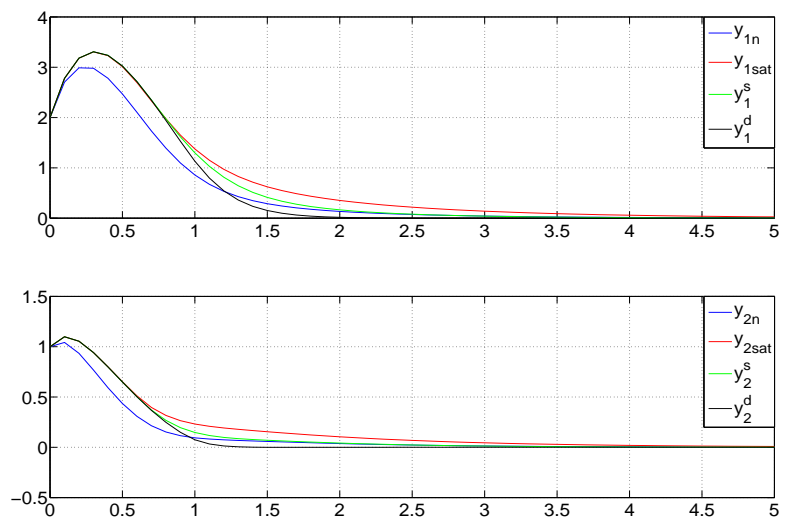

Fig. 1. System outputs

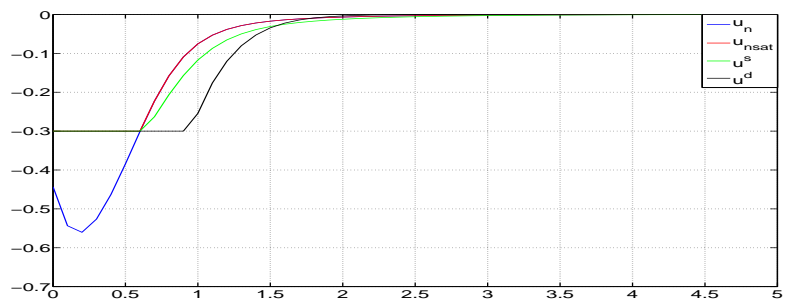

Fig. 2. Control input

$$
D_{1}^{c}=\left(\begin{array}{ll}
-0.33 & -0.27
\end{array}\right), D_{2}^{c}=\left(\begin{array}{ll}
-0.13 & -0.89
\end{array}\right)
$$

For the proposed example, the control goal is to ensure the state trajectory convergence to the origin in spite of the input saturation. One can observe from the depicted figures that the results are slightly better for the dynamic controller with the proposed T-S approach. It can be noted that using the dynamic controller allows to decrease the radius of the ball in which the state convergence is ensured. Moreover, the numerical results are $\beta_{s}=15.09$ and $\beta_{d}=9.46$. To analyse the results in the four situations, the following comparison criteria are selected: the fall time of the state variables $x_{1}$ and $x_{2}$ denoted $t_{1}$ and $t_{2}$ respectively that are to be minimised, the energy of each output denoted $E_{y_{1}}$ and $E_{y_{2}}$ respectively $\left(E_{y_{i}}=\int_{0}^{t} y_{i}^{2}(t) d t\right)$ to be minimized; the contol input energy denoted $\left.E_{u}\left(E_{u}=\int_{0}^{t} u^{T}(t) u(t) d t\right)\right)$. The results are displayed in the following table, where case 1 refers to the nominal closed loop system without saturation, case 2 refers to nominal closed loop system with saturation, case 3 and case 4 respectively refer to the proposed static and dynamic T-S approach. The proposed T-S controller design

\begin{tabular}{|c|c|c|c|c|}
\hline & case 1 & case 2 & case 3 & case 4 \\
\hline$t_{1}$ & $2 s$ & $2.8 s$ & $2.1 s$ & $1.6 s$ \\
\hline$t_{2}$ & $1 s$ & $1.8 s$ & $1.4 s$ & $0.91 s$ \\
\hline$E_{y 1}$ & 278.06 & 394.65 & 382.12 & 369.04 \\
\hline$E_{y 2}$ & 21.57 & 31.05 & 29.22 & 28.70 \\
\hline$E_{u}$ & 8.17 & 3.64 & 3.99 & 5.11 \\
\hline
\end{tabular}

approach not only ensures the stability of the closed-loop system, but also compensate the saturation effect by adding more energy to the control input.

\section{CONCLUSIONS}

Using the T-S approach to describe both the nonlinearities and the input saturation, a nonlinear system with saturated actuator(s) can be represented with a T-S model. This unified representation allows to deal with these difficulties and to synthesize an output feedback controller which gains depend on the saturation bounds. The solution of this problem is based on the Lyapunov theory using the descriptor approach and is expressed in terms of LMI. Two output feedback controllers were synthetized, a static controller and a dynamic one. The dynamic controller was considered in order to increase the number of degrees of freedom in the design and improve the control performances.

\section{REFERENCES}

[1] S. Bezzaoucha, B. Marx, D. Maquin, and J. Ragot. Linear feedback control input under actuator saturation : A Takagi-Sugeno approach. In 2nd International Conference on Systems and Control (ICSC'12), Marrakech, Morocco, 2012.

[2] K. Guelton, T. Bouarar, and N. Manamanni. Robust dynamic output feedback fuzzy Lyapunov stabilization of Takagi-Sugeno systems- a descriptor redundancy approach. Fuzzy Sets and Systems, 160(19):2796-2811, 2009.

[3] T.M. Guerra, A. Kruszewski, L. Vermeiren, and H. Tirmant. Conditions of output stabilization for nonlinear models in the TakagiSugeno's form. Fuzzy Sets and Systems, 157(9):1248-1259, 2006.

[4] V. Kapila and K. Grigoriadis. Actuator Saturation Control. Marcel Dekker, Inc. New York, 2002.

[5] N. Kapoor, A. R. Teel, and P. Daoutidis. An anti-windup design for linear systems with input saturation. Automatica, 34(5):559-574, 1998.

[6] Y-M. Ma and G-H. Yang. Performance analysis for linear discretetime systems subject to actuator saturation. In 2008 American Control Conference, Washington, USA, 2008.

[7] B. Marx, D. Koenig, and D. Georges. Robust fault tolerant control for descriptor systems. IEEE Transactions on Automatic Control, 49(10):1869-1875, 2004.

[8] B. Marx, D. Koenig, and J. Ragot. Design of observers for TakagiSugeno descriptor systems with unknown inputs and application to fault diagnosis. IET Control Theory and Applications, 1(5):14871495, 2007.

[9] T. Takagi and M. Sugeno. Fuzzy identification of systems and its applications to modeling and control. IEEE Transactions on Systems, Man, and Cybernetics, 15(1):116-132, 1985.

[10] K. Tanaka, H. Ohtake, and H.O. Wang. A descriptor system approach to fuzzy control system design via fuzzy Lyapunov functions. IEEE Transactions on Fuzzy Systems, 15(3):333-341, 2007.

[11] K. Tanaka and H.O. Wang. Fuzzy Control Systems Design and Analysis: A Linear Matrix Inequality Approach. John Wiley \& Sons, Inc., 2001.

[12] S. Tarbouriech, G. Garcia, J.M. Gomes da Silva Jr., and I. Queinnec. Stability and Stabilization of Linear Systems with Saturating Actuators. Springer-Verlag, 2011.

[13] S. Tong and W. Wang. Decentralized robust control for uncertain T-S fuzzy large-scale systems with time-delay. International Journal of Innovative Computing Information and Control, 3(3):657-672, 2007.

[14] W. Xie. Improved $\mathscr{L} 2$ gain performance controller synthesis for Takagi-Sugeno fuzzy system. IEEE Transactions on Fuzzy Systems, 16(5):1142-1150, 2008.

[15] L. Zaccarian and A.R. Teel. A common framework for anti-windup, bumpless transfer and reliable design. Automatica, 38(10):1735-1744, 2002.

[16] K. Zhang, B. Jiang, and P. Shi. A new approach to observer-based fault-tolerant controller design for Takagi-Sugeno fuzzy systems with state delay. Circuits, systems, and signal processing, 28(5):679-697, 2009 